\title{
A fast incremental-iterative procedure for ultimate strength analysis of composite cross-sections of arbitrary shape
}

\author{
C. G. Chiorean \\ Technical University of Cluj-Napoca, Faculty of Civil Engineering, \\ Romania
}

\begin{abstract}
A new computer method for bi-axial ultimate strength analysis of composite steel-concrete cross-sections of arbitrary shape subjected to axial force and biaxial bending moments is developed. An incremental-iterative procedure based on arc-length approach is proposed in order to determine, in a unitary formulation, both interaction diagrams and moment capacity contours, overcoming the difficulties and inaccuracies of the previously published methods. This procedure adopts a tangent stiffness strategy for the solution of the non-linear equilibrium equations thus resulting in a high rate and unconditionally convergence. An object oriented computer program, to obtain the ultimate strength of composite cross-sections under combined biaxial bending and axial load was developed. Examples run and comparisons made have proved the effectiveness and time saving of the proposed method of analysis.

Keywords: composite cross-sections, ultimate strength, arc-length method, bi-axial bending.
\end{abstract}

\section{Introduction}

In recent years, some methods have been presented for the ultimate strength analysis of various concrete and composite steel-concrete sections such as rectangular, $\mathrm{L}$ and $\mathrm{T}$-shape, polygonal and circular under biaxial moments and axial loads [1-5]. Among several existing techniques, two are the most common; the first consists of a direct generation of points of the failure surface by varying the position and inclination of the neutral axis and imposing a strain distribution corresponding to a failure condition. This technique generates the failure surface 
through 3D curves (Fig. 1) that are difficult to plot, having limited applicability in the practical applications. The second approach is based upon the solution of the non-linear equilibrium equations according to the classical Newton's scheme to obtain either the strain equilibrium plane or the location and inclination of the neutral axis. In general, these methods generates plane interaction curves and gives fast solutions but are sensitive to the origin of the loading axes and some problems in convergence may arise, particularly when the initial or starting values of variables are not selected properly and under large axial forces [2]. These methods can be further distinguished by how they plot the interaction curves. These curves may be computed, indirectly, by explicit computation of the moment-curvature response and the failure of the cross-section corresponds to the top of the moment-curvature diagram when any of the materials reaches its predefined maximum allowable strain $[3,5]$. This method can be used under any loading mode, but is rather time-consuming and the basic equations of equilibrium are not always satisfied [3]. On the other hand, to determine directly the interaction diagrams or load contours, the exact location of the neutral axis may be determined by solving three coupled nonlinear system of equations by an iterative approach $[1,2,4]$.

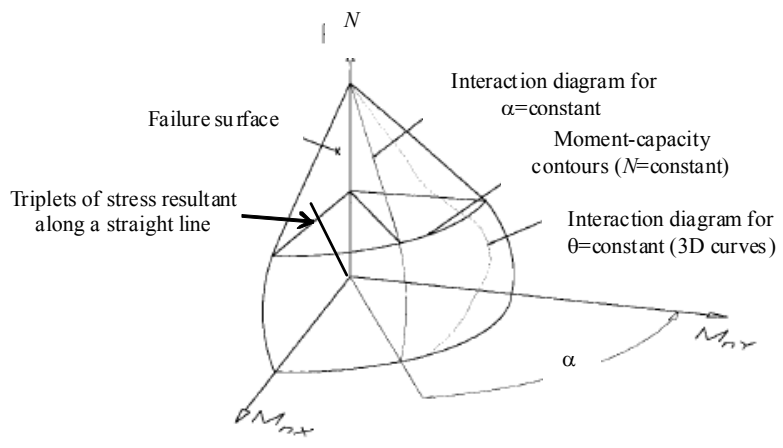

Figure 1: $\quad$ Failure surface and interaction diagrams.

These algorithms are not straightforward to implement, starting or initial values are not always simply to choose, and problems of convergence may arise especially when strain softening of the concrete in compression is taken into account, and they may become unstable near the state of the pure compression. There exist three different methods to generate plane interaction curves for crosssections under biaxial bending: (1) interaction curves for a given bending moment ratio [1], (2) load contours for a given axial load [2] and (3) generate triplets of stress-resultants on the failure surface by extending an arbitrary oriented straight line [4]. The plane interaction curves generated by these methods are depicted in Fig. 1.

The main objective of the present paper is to present a new formulation by which the biaxial interaction diagrams and moment capacity contours of an arbitrary composite cross-section can be determined. Essentially, the inelastic 
cross-section analysis method employed herein uses the accuracy of the fibber element analysis through the use of path integral approach for numerical integration of the cross-sectional nonlinear characteristics, and addresses its efficiency and modelling shortcomings both to failure surface generation procedure, overcoming the difficulties and inaccuracies of the previously proposed methods, and to postprocessing procedure of the axial force and bending moments obtained at a cross-section level, in order to check directly that they fulfil the ultimate limit state condition.

\section{Mathematical formulation}

\subsection{Assumptions and problem definition}

Consider the arbitrary cross-section shape subjected to the action of the external bending moments about each global axes and axial force as shown in Figure 2. It is assumed that plane section remains plane after deformation. This implies perfect bonding between the steel and concrete components of a composite cross section. Thus resultant strain distribution corresponding to the curvatures about global axes $\Phi=\left\{\phi_{x}, \phi_{y}\right\}$ and the axial compressive strain $\varepsilon_{0}$ can be expressed at a generic point $(x, y)$ in a linear form as:

$$
\varepsilon=\varepsilon_{0}+\phi_{x} y+\phi_{y} x
$$

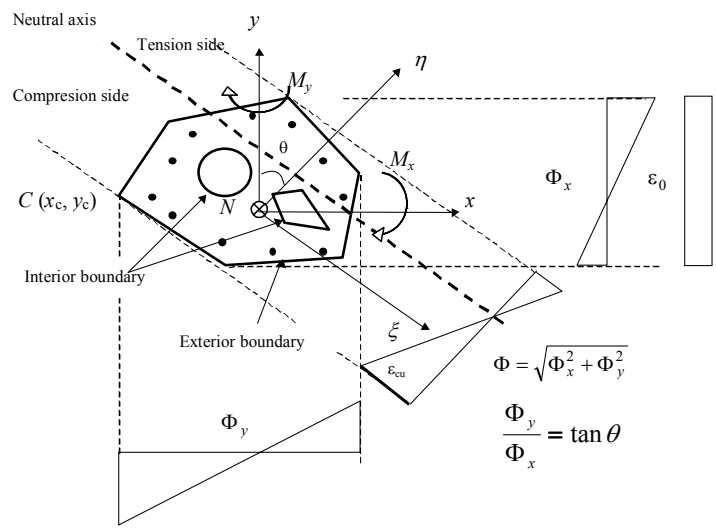

Figure 2: Model of arbitrary composite cross-section.

The constitutive relations for concrete under compression are represented by a combination of a second-degree parabola and a straight line as depicted in Fig. 3(a). The parameter $\gamma$ represents the degree of confinement in the concrete, and allows for the modelling of creep and confinement in the concrete by simply varying the crushing strain $\varepsilon_{c 0}$ and $\gamma$ respectively. The tensile strength of concrete is neglected. A multi-linear elasto-plastic stress-strain relationship, both in tension and in compression, is assumed for the structural steel and the steel 
reinforcing bars (Fig. 3(b)). At ultimate strength capacity the equilibrium is satisfied when the external forces are equal to the internal ones and in the most compressed or tensioned point the ultimate strain is attained. These conditions can be represented mathematically in terms of the following nonlinear system of equations as:

(a)

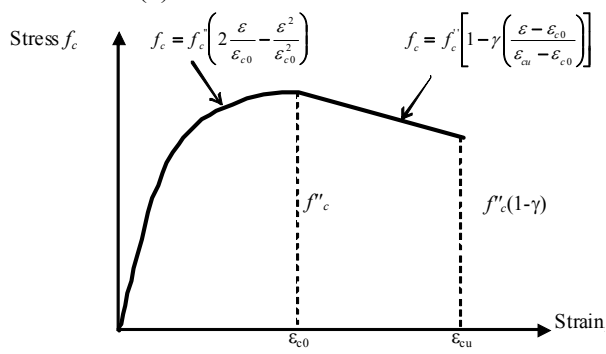

(b)

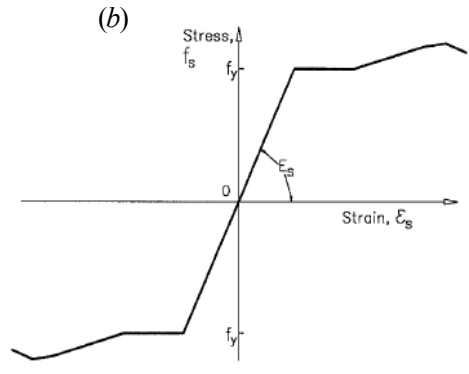

Figure 3: $\quad$ Stress-strain relationships: (a) for concrete in compression; $(b)$ for steel.

$$
\left\{\begin{array}{l}
\int_{A} \sigma\left(\varepsilon_{0}, \phi_{x}, \phi_{y}\right) d A-N=0 ; \int_{A} \sigma\left(\varepsilon_{0}, \phi_{x}, \phi_{y}\right) y d A-M_{x}=0 ; \int_{A} \sigma\left(\varepsilon_{0}, \phi_{x}, \phi_{y}\right) x d A-M_{y}=0 \\
\varepsilon_{0}+\phi_{x} y_{c}\left(\phi_{x}, \phi_{y}\right)+\phi_{y} x_{c}\left(\phi_{x}, \phi_{y}\right)-\varepsilon_{u}=0
\end{array}\right.
$$

and in which $N, M_{x}, M_{y}, \varepsilon_{0}, \phi_{x}, \phi_{y}$ represent the unknown. In the Eqs. (2) the first three relations represent the basic equations of equilibrium for the axial load $N$ and the biaxial bending moments $M_{x}, M_{y}$ respectively, given in terms of the stress resultants. The last equation represents the ultimate strength capacity condition; that is, in the most compressed or most tensioned point the ultimate strain is attained, and in which $x_{c}\left(\phi_{x}, \phi_{y}\right)$ and $y_{c}\left(\phi_{x}, \phi_{y}\right)$ represent the coordinates of the point in which this condition is imposed. The coordinates of the "constrained" point can be always determined for each inclination of the neutral axis defined by the parameters $\phi_{x}$ and $\phi_{y}$, and $\varepsilon_{u}$ represents the ultimate strain either in most compressed concrete point or in most tensioned reinforcement steel fibre. Under the above assumptions, the problem of the ultimate strength analysis of composite cross-sections can be formulated as: With strain distribution corresponding to a failure condition, find the ultimate resistances $N$, $M_{x}, M_{y}$ so as to fulfil the basic equations of equilibrium and one of the following linear constraints:

$$
\text { (a) }\left\{\begin{array}{l}
L_{1}\left(N, M_{x}, M_{y}\right) \equiv M_{x}-M_{x 0}=0 \\
L_{2}\left(N, M_{x}, M_{y}\right) \equiv M_{y}-M_{y 0}=0
\end{array} ;(b)\left\{\begin{array}{l}
L_{1}\left(N, M_{x}, M_{y}\right) \equiv N-N_{0}=0 \\
L_{2}\left(N, M_{x}, M_{y}\right) \equiv M_{x}-M_{x 0}=0
\end{array}\right.\right.
$$

where $N_{0}, M_{x 0}, M_{y 0}$ represents the given axial force and bending moments, respectively. 

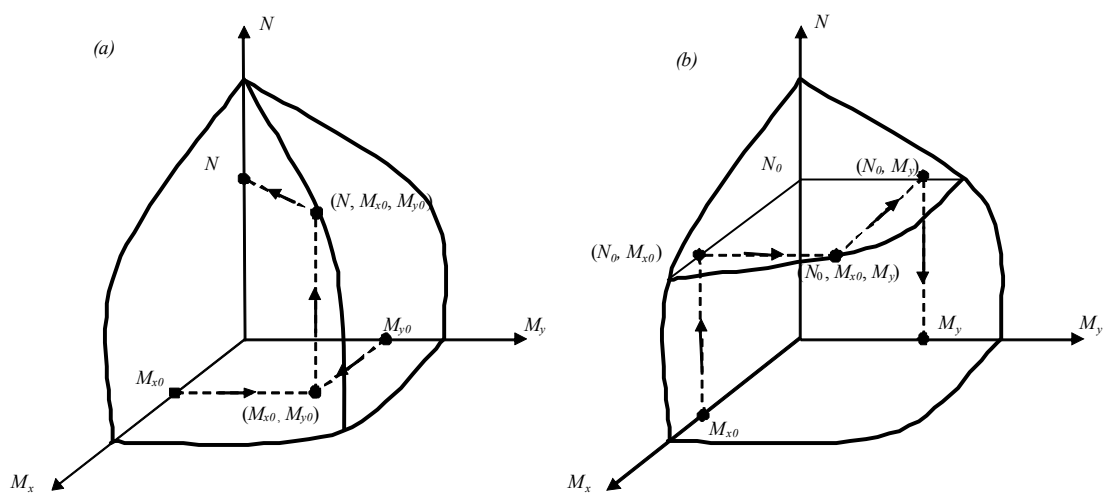

Figure 4: General solution procedures. (a) Interaction diagrams for given bending moments; $(b)$ Moment-capacity contours for given axial force and bending moment $M_{x}$.

The general solution procedure is organized around solving the nonlinear system (2) for one of two linear constraints defined by the Eqs. $(3 a, b)$ Corresponding to each linear constraints we can define a point on the failure surface as: (I) when the constraints $(a)$ are injected in the nonlinear system (2), a point on the failure surface is defined computing the axial resistance $N$ associated to a failure criterion and for a fixed value of bending moments $\left(M_{x}\right.$, $M_{y}$ ); (II) when constraints $(b)$ are used, the point is associated to a fixed axial load $(N)$ and a given bending moment $M_{x}$ about $x$ axis. All these situations are graphically illustrated in Figure 4.

\subsection{Method of solution}

An incremental-iterative procedure based on arc-length constraint equation is proposed in order to determine the biaxial strength of an arbitrary composite steel-concrete cross section accordingly to the already described situations. The failure diagrams corresponds either to maximum strains attained at the outer compressed point of the concrete section (i.e. $\varepsilon_{u}$ equal to the compressive strain at failure) or to maximum strains attained in most tensioned reinforcement steel fibre (i.e. $\varepsilon_{u}$ equal to the tensile steel strain at failure). Consider an irregular composite section as shown in Figure 2. The global $x, y$-axes of the cross section could have their origin either in elastic or plastic centroid of the cross-section. For each inclination of the neutral axis defined by the parameters $\phi_{x}$ and $\phi_{y}$ the farthest point on the compression side (or the most tensioned steel bar position) is determined (i.e. the point with co-ordinates $x_{c}, y_{c}$ ). We assume that at this point the failure condition is met, and consequently the axial compressive strain $\varepsilon_{0}$ can be expressed as:

$$
\varepsilon_{0}=\varepsilon_{u}-\left(\phi_{x} y_{c}+\phi_{y} x_{c}\right)
$$


Thus, resultant strain distribution corresponding to the curvatures $\phi_{x}$ and $\phi_{y}$ can be expressed in linear form as:

$$
\varepsilon\left(\phi_{x}, \phi_{y}\right)=\varepsilon_{u}+\phi_{x}\left(y-y_{c}\right)+\phi_{y}\left(x-x_{c}\right)
$$

In this way, substituting the strain distribution given by the Eq. (5) in the basic equations of equilibrium, the unknown $\varepsilon_{0}$ together with the failure constraint equation can be eliminated from the nonlinear system (2). Thus the basic equations of equilibrium together with the linear constraints Eqs. (3(a)) (3(b)) forms a determined nonlinear system of equations (i.e. 5 equations and 5 unknowns):

$$
\left\{\begin{array}{l}
\int_{A} \sigma\left(\varepsilon\left(\phi_{x}, \phi_{y}\right)\right) d A-N=0 \\
\iint_{A} \sigma\left(\varepsilon\left(\phi_{x}, \phi_{y}\right)\right) y d A-M_{x}=0+\left\{\begin{array}{l}
L_{1}\left(N, M_{x}, M_{y}\right)=0 \\
L_{2}\left(N, M_{x}, M_{y}\right)=0
\end{array}\right. \\
\int_{A} \sigma\left(\varepsilon\left(\phi_{x}, \phi_{y}\right)\right) x d A-M_{y}=0
\end{array}\right.
$$

and the solutions can be obtained iteratively following an approach outlined in the next sections.

\subsubsection{Interaction diagrams for given bending moments}

In this case introducing the constraints $(3 a)$ in the system (6) the problem of the ultimate strength analysis of cross-section can be expressed mathematically as:

$$
\int_{A} \sigma\left(\delta\left(\phi_{x}, \phi_{y}\right)\right) d A-N=0 ; \int_{A} \partial\left(\delta\left(\phi_{x}, \phi_{y}\right)\right) y d A-\lambda M_{x 0}=0 ; \int_{A} \partial\left(\delta\left(\phi_{x}, \phi_{y}\right)\right) x d A-\lambda M_{y 0}=0
$$

in which axial load $N$ and curvatures $\phi_{x}$ and $\phi_{y}$ represents the unknowns and $\lambda$ represents the load parameter defining the intensity of the bending moments. If we regard the curvatures as independent variables in axial force equation, the curvatures and the load amplifier factor $\lambda$ are given by solving the following nonlinear system of equations:

$$
\left\{\begin{array}{l}
\int_{A} \sigma\left(\varepsilon\left(\phi_{x}, \phi_{y}\right)\right) y d A-\lambda M_{x 0}=0 \\
\int_{A} \sigma\left(\varepsilon\left(\phi_{x}, \phi_{y}\right)\right) x d A-\lambda M_{y 0}=0
\end{array}\right.
$$

This can be rewritten in terms of non-linear system of equations in the following general form:

$$
\mathbf{F}(\lambda, \boldsymbol{\Phi})=\mathbf{f}^{\mathrm{int}}-\lambda \mathbf{f}^{e x t}=\mathbf{0}
$$

To traverse a solution path a proper parametrization is needed. A common setting of a continuation process is to augment the equilibrium equations (9) with a constraint [6]. In this case the curvature-moment constraint can be defined by equation $g$ in the following form:

$$
\mathbf{H}(\lambda, \boldsymbol{\Phi})=\left\{\begin{array}{l}
\mathbf{f}^{i n t}-\lambda \mathbf{f}^{e x t}=\mathbf{0} \\
g(\lambda, \boldsymbol{\Phi})=0
\end{array}\right.
$$


In this procedure, commonly called arc-length method, these equations are solved in a series of steps or increments, usually starting from the unloaded state $(\lambda=0)$, and the solution to (10) is referred to as equilibrium path. Instead of solving Eqs. (10) directly, an indirect solution scheme for the constraint equation may be introduced. According to the indirect arc-length technique [6], the iterative changes of curvature vector $\delta \Phi$ for the new unknown load level $\Delta \lambda_{k+1}=\Delta \lambda_{k}+\delta \lambda$, is written as:

$$
\delta \mathbf{\Phi}=-\mathbf{K}_{T}{ }^{-1} \mathbf{F}+\delta \lambda \mathbf{K}_{T}{ }^{-1} \mathbf{f}^{e x t}=\delta \mathbf{F}+\delta \lambda \delta \mathbf{\Phi}_{T}
$$

where $\mathbf{F}$ represents the out-of-balance force vector (Eq. 9) and $\mathbf{K}_{T}$ represents the tangent stiffness matrix of the cross-section:

$$
\mathbf{K}_{T}=\left(\frac{\partial \mathbf{F}}{\partial \boldsymbol{\Phi}}\right)=\left[\begin{array}{cc}
\frac{\partial M_{x}{ }^{i n t}}{\partial \phi_{x}} & \frac{\partial M_{x}{ }^{i n t}}{\partial \phi_{y}} \\
\frac{\partial M_{y}{ }^{i n t}}{\partial \phi_{x}} & \frac{\partial M_{y}{ }^{i n t}}{\partial \phi_{x}}
\end{array}\right]
$$

in which the partial derivatives are with respect to the strains and stresses evaluated at current iteration $k$. Assuming the strain distribution given by the Eq.(5), the coefficients of the stiffness matrix can be symbolically evaluated as:

$$
\begin{aligned}
& k_{11}=\frac{\partial M_{x}^{\text {int }}}{\partial \phi_{x}}=\frac{\partial}{\partial \phi_{x}} \int_{A} \sigma\left(\varepsilon\left(\phi_{x}, \phi_{y}\right)\right) y d A=\int_{A} \frac{\partial \sigma}{\partial \varepsilon} \frac{\partial \varepsilon}{\partial \phi_{x}} y d A=\int_{A} E_{T} y\left(y-y_{c}\right) d A \\
& k_{12}=\frac{\partial M_{x}^{i n t}}{\partial \phi_{y}}=\frac{\partial}{\partial \phi_{y}} \int_{A} \sigma\left(\varepsilon\left(\phi_{x}, \phi_{y}\right)\right) y d A=\int_{A} \frac{\partial \sigma}{\partial \varepsilon} \frac{\partial \varepsilon}{\partial \phi_{y}} y d A=\int_{A} E_{T} y\left(x-x_{c}\right) d A \\
& k_{21}=\frac{\partial M_{y}^{\text {int }}}{\partial \phi_{x}}=\frac{\partial}{\partial \phi_{x}} \int_{A} \sigma\left(\varepsilon\left(\phi_{x}, \phi_{y}\right)\right) x d A=\int_{A} \frac{\partial \sigma}{\partial \varepsilon} \frac{\partial \varepsilon}{\partial \phi_{x}} x d A=\int_{A} E_{T} x\left(y-y_{c}\right) d A \\
& k_{22}=\frac{\partial M_{y}^{\text {int }}}{\partial \phi_{y}}=\frac{\partial}{\partial \phi_{y}} \int_{A} \sigma\left(\varepsilon\left(\phi_{x}, \phi_{y}\right)\right) x d A=\int_{A} \frac{\partial \sigma}{\partial \varepsilon} \frac{\partial \varepsilon}{\partial \phi_{y}} x d A=\int_{A} E_{T} x\left(x-x_{c}\right) d A
\end{aligned}
$$

where the coefficients $k_{\mathrm{ij}}$ are expressed in terms of the tangent modulus of elasticity $E_{t .}$. Thus the incremental curvatures for the next iteration can be written as:

$$
\Delta \boldsymbol{\Phi}_{k+1}=\Delta \boldsymbol{\Phi}_{k}+\delta \boldsymbol{\Phi}
$$

This procedure is iterated until convergence upon a suitable norm is attained. Assuming that a point $\left({ }^{\tau} \Phi,{ }^{\tau} \lambda\right)$ of the equilibrium path has been reached, the next point $\left({ }^{\tau+\Delta \tau} \Phi,{ }^{\tau+\Delta \tau} \lambda\right)$ of the equilibrium path is then computed updating the loading factor and curvatures as:

$$
\begin{aligned}
&{ }^{\tau+\Delta \tau} \lambda={ }^{\tau} \lambda+\Delta \lambda_{k+1} \\
&{ }^{\tau+\Delta \tau} \boldsymbol{\Phi}={ }^{\tau} \boldsymbol{\Phi}+\Delta \boldsymbol{\Phi}_{k+1}
\end{aligned}
$$

In this way with curvatures and loading factor known, the axial force resistance $N$ is computed based on the resultant strain distribution corresponding to the curvatures $\phi_{x}$ and $\phi_{y}$ through Equation (7), and the ultimate bending moments, $M_{x}$ and $M_{y}$, are obtained scaling the reference external moments $M_{x 0}$ and $M_{y 0}$ through current loading factor $\lambda$ given by Equation (15). Graphical 
representation of the present method according to Equation (10) is depicted in Figure 5. It is important to note that, the stiffness matrix of cross-section $K_{T}$ given by the Equations (13), could become negative definite or singular during the iterative process therefore the above procedure based on arc-length constraint equation is essential to overcome these difficulties.
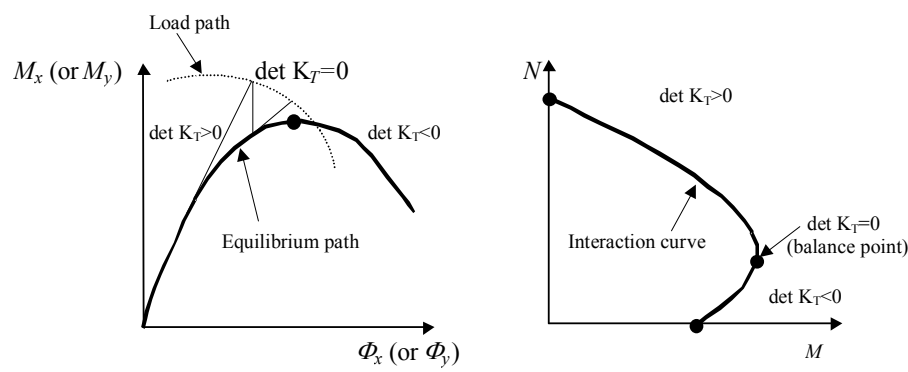

Figure 5: Geometrical representation of the present method.

\subsubsection{Moment capacity contour for given axial force $N$ and bending moment $M_{x}$}

In this case, injecting the linear constraints $(3 b)$ in the nonlinear system (6), and arranging the system accordingly with the decoupled unknowns, we obtain:

$$
\left\{\begin{array}{l}
\int_{A} \sigma\left(\varepsilon\left(\phi_{x}, \phi_{y}\right)\right) d A-N_{0}=0 \\
\int_{A} \sigma\left(\varepsilon\left(\phi_{x}, \phi_{y}\right)\right) y d A-\lambda M_{x 0}=0
\end{array}+\int_{A} \sigma\left(\varepsilon\left(\phi_{x}, \phi_{y}\right)\right) x d A-M_{y}=0\right.
$$

in which bending moment $M_{y}$ and curvatures $\phi_{x}$ and $\phi_{y}$ represents the unknowns. Following a similar approach as presented above, the curvatures are obtained solving the first two equations and then with this strain distribution the bending moment resistance about $y$ axis is computed with the last equation of the system.

\subsection{Evaluation of tangent stiffness and stress resultant}

Based on Green's theorem, the integration of the stress resultant and stiffness coefficients over the cross-section will be transformed into line integrals along the perimeter of the cross-section. For this purpose, is necessary to transform the variables first, so that the stress field is uniform in a particular direction, given by the current position of the neutral axis [7]. This is achieved by rotating the reference axes $x, y$ to $\xi, \eta$ oriented parallel to and perpendicular to the neutral axis, respectively as shown in Fig. 2. Thus, the stress field is uniform in direction parallel with the neutral axis and strains and the internal forces carried on by the compressive concrete and structural steel can be obtained by the following expressions: 


$$
\begin{aligned}
& N_{\text {int }}=\iint \sigma(x, y) d x d y=\iint \sigma(\eta) d \xi d \eta \\
& M_{x, \text { int }}=\iint \sigma(x, y) y d x d y=\iint \sigma(\eta)(-\xi \sin \theta+\eta \cos \theta) d \xi d \eta=M_{\xi, \text { int }} \cos \theta-M_{\eta, \text { int }} \sin \theta \\
& M_{y, \text { int }}=\iint \sigma(x, y) x d x d y=\iint \sigma(\eta)(\xi \cos +\eta \sin \theta) d \xi d \eta=M_{\xi, \text { int }} \sin \theta-M_{\eta, \text { int }} \cos \theta
\end{aligned}
$$

where $N_{\text {int }}, M_{\xi, \text { int }}$ and $M_{\eta, \text { int }}$ are the internal axial force and bending moments about the $\xi$ and $\eta$ axis respectively and can be computed based on the Green's path integral approach. The tangent stiffness matrix coefficients are computed in the same way. In order to perform the integral of a determined side of the contour, polygonal or circular, of the integration area, the interpolatory GaussLobatto method is used. Though this rule has lower order of accuracy than customary Gauss-Legendre rule, it has integration points at each ends of interval, and hence performs better in detecting yielding. However, because the stress filed is defined by a step function and there is no continuity in the derivative, the polynomial interpolation can produce important integration errors. In this case, an adaptive quadrature strategy can be applied. In this context of the adaptivity quadratures, the Lobatto integration scheme has another advantage over the Legendre integration scheme, observing that the point corresponding to the left end in one interval is the same as the point corresponding to right end in the next. Consequently, the cost of evaluating a Lobatto rule is reduced by about one integrand evaluation comparing with Legendre rule. The steel bars are assumed discrete points with area $A_{s j}$, co-ordinates $x_{s j}, y_{s j}$ and stress $f_{s j}$.

\section{Computational example}

Based on the analysis algorithm just described, a computer program ASEP has been developed to study the biaxial strength behaviour of arbitrary concrete-steel cross sections. In order to demonstrate the validity, accuracy, unconditionally convergence and time saving of the analytic procedure developed here, the interaction diagrams and moment capacity contours of a rectangular crosssection with asymmetrically placed structural steel (Fig. 6(a)) are determined and compared with the numerical procedure developed in [2].

Characteristic strength for concrete in compression is $f_{c}=31.79 \mathrm{Mpa}$ and the stress-strain curve which consists of a parabolic and linear-part was used in the calculation, with the crushing strain $\varepsilon_{0}=0.002$ and ultimate strain $\varepsilon_{c u}=0.0035$. The Young modulus for all steel sections was $200 \mathrm{GPa}$ while the maximum strain was $\varepsilon_{s u}= \pm 1 \%$. The yield strength of steel reinforcing bars is $f_{y}=420 \mathrm{MPa}$, whereas for the structural steel the following values has been considered $f_{\text {y,flange }}=255 \mathrm{MPa}$ $f_{y, w e b}=239 \mathrm{MPa}$. In order to demonstrate the unconditionally convergence of the algorithms developed in the current paper the cross-section has been analysed, drawing the interaction diagrams and moment capacity contours for axial loads near the axial load capacity, considering both geometric and plastic centroid of the cross-section. Convergence problems have been experienced by the Chen et al. [2] in this portion of the moment capacity contour when the geometrical centroid of the cross-section has been taken as the reference loading axes. 
Figure $6(b)$ presents the moment capacity contours, obtained by the present algorithm and those obtained by the Chen et al. [2] for axial load $N=3000 \mathrm{kN}$, considering as reference loading axes, geometric (GC) and plastic centroid (PC) respectively.

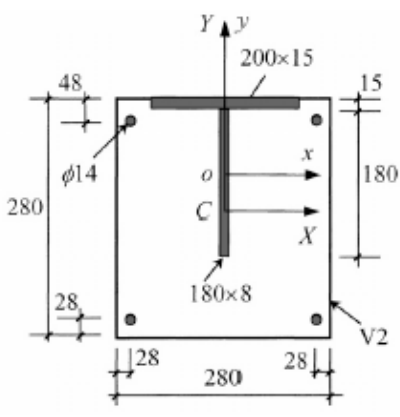

(a)

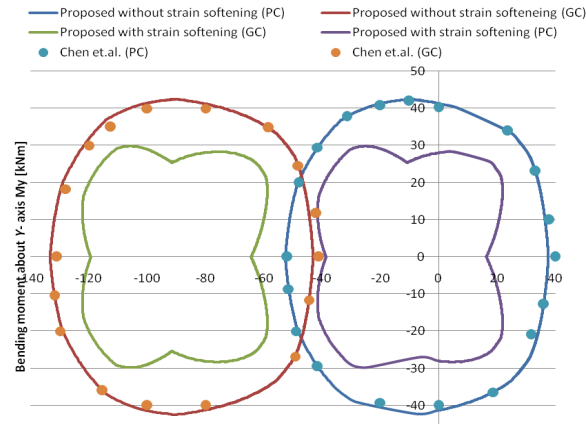

(b)

Figure 6: (a) Rectangular section with asymmetrically placed structural steel.

(b) Moment capacity contour with axial load $N=3000 \mathrm{kN}$.

No convergence problems have been experienced by the proposed approach, even when the geometric centroid has been chosen as reference axes or the strain-softening of the concrete in compression has been taken into account $(\gamma=0.15)$, and a maximum of just two iterations has been required during the incremental-iterative process. As it can be seen the results obtained in the current paper and those reported in [2] agree closely in both cases. The effect of the strain-softening is not taken into account in the method proposed in [2]. However it is important to note that, although claimed, the method proposed by Chen does not generate genuinely plane moment-capacity curves. The method proposed in [2] fails in some circumstances to draw the moment capacity contour, under a fixed axial load, and in order to overcome some divergences, axial load value is slightly adjusted. Figure 7(a) shows the complete interaction diagrams under uniaxial bending moment about $x$ axis with and without the effect of the strain softening of the concrete. As it can be seen near the axial load capacity multiple solutions exists in the $N-M$ space when the strain softening is modelled. This can be clearly observed on the moment-ultimate curvature diagrams (Fig. 7(b)), when multiple "snap-through" phenomenons occur in these situations. When the strain-softening is ignored $(\gamma=0)$, the moment-ultimate curvature curve is flat in that region (Fig.7.b) that explains the uniqueness of the solution in the $N-M$ space in this case (Fig.7(a)). Figure 8 shows the comparative interaction diagrams for $\alpha=15^{\circ}$ and $30^{\circ}$ respectively $\left(M_{y}=\tan (\alpha) M_{x}\right)$. The bending moments are computed about axes $x-y$, which pass through the geometric centroid of the cross-section. 

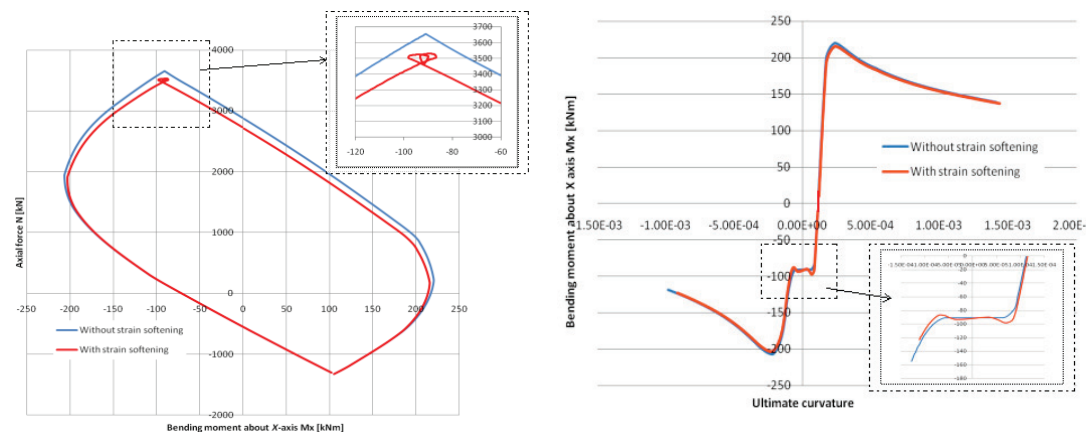

Figure 7: (a) Interaction diagrams under uniaxial bending moment about $x$ axis; (b) Bending moment-ultimate curvature variation.

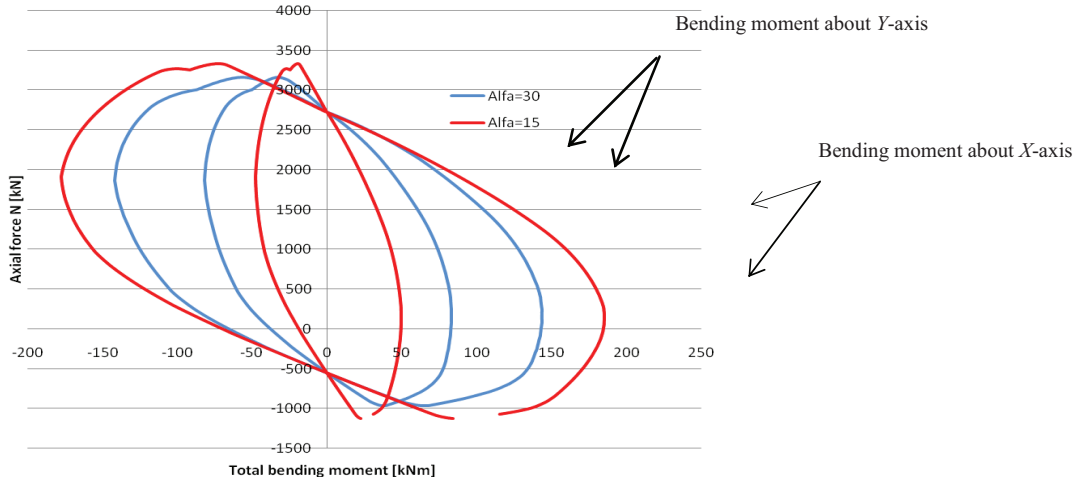

Figure 8: Biaxial interaction diagrams.

\section{Conclusions}

A new computer method based on incremental-iterative arc-length technique has been presented for the ultimate strength analysis of composite steel-concrete cross-sections subjected to axial force and biaxial bending. Comparing the algorithm presented in the current paper with the existing methods it can be concluded that the proposed approach is general and complete, can determine both interaction diagrams and moment capacity contours, and, of great importance, it is fast, the diagrams are directly calculated by solving, at a step, just two coupled nonlinear equations, and assures convergence for any load case, even near the state of pure compression or tension and is not sensitive to the initial/starting values, how the origin of the reference loading axes is chosen or to the strain softening effect for the concrete in compression. Furthermore, the proposed method as is formulated can be applied to provide directly the ultimate resistances of the cross-section, supposing that one or two components of the section forces are known, without the need of knowing in advance the whole 
interaction diagram or moment capacity contour. The method has been verified by comparing the predicted results with the established results available from the literature. It can be concluded that the proposed numerical method proves to be reliable and accurate for practical applications in the design of composite steelconcrete beam-columns and can be implemented in the advanced analysis techniques of $3 \mathrm{D}$ composite frame structures.

\section{Acknowledgement}

The writer gratefully acknowledges the support from Romanian Research Foundation (CNCSIS- Grant PNII-IDEI No. 193/2008) for this study.

\section{References}

[1] Rodrigues, J.A. \& Aristizabal-Ochoa, J.D. Biaxial interaction diagrams for short RC columns of any cross section, Journal of Structural Engineering, ASCE, 125(6): 672-683, 1999.

[2] Chen S.F., Teng J.G., Chan S.L. Design of biaxially loaded short composite columns of arbitrary section, Journal of Structural Engineering, ASCE, 127(6), 678-685, 2001.

[3] Sfakianakis, M.F., Biaxial bending with axial force of reinforced, composite and repaired concrete cross sections of arbitrary shape by fibber model and computer graphics, Advances in Engineering Software, 33, 227-242, 2002.

[4] Rosati, L., Marmo, F., Serpieri, R., Enhanced solution strategies for the ultimate strength analysis of composite steel-concrete sections subject to axial force and biaxial bending, Computer methods in applied mechanics and engineering, 197(9-12), 1033-1055, 2008.

[5] Charalampakis, A.E., Koumousis, V.K., Ultimate strength analysis of composite sections under biaxial bending and axial load, Advances in Engineering Structures, 39(11), 923-936, 2008.

[6] Crisfield, M.A. Non linear finite element analysis of solids and structures, Wiley, Chichester, 1991.

[7] Chiorean, C.G., A fast incremental-iterative procedure for inelastic analysis of RC cross-sections of arbitrary shape, Acta Technica Napocensis, 47, 8598, 2004. 